\title{
Organic Transparent Electrodes Applied to Polymer Light Emitting Diodes*
}

\author{
Wataru Mizutani ${ }^{\dagger}$ \\ Nanosystem Research Institute, National Institute of Advanced Industrial Science and Technology (AIST), \\ Higashi 1-1-1, Tsukuba, Ibaraki 305-8562, Japan
}

Kazumi Aoba, Hideki Sakai, Takashi Ohmori, and Hayato Hyakutake Denshi Kako, Co.,LTD, 3309 Shimokoyama, Shimotsuke, Tochigi 329-0502, Japan (Received 15 December 2013; Accepted 5 February 2014; Published 22 February 2014)

\begin{abstract}
Transparent electrodes are widely used in touch panels, solar cells, displays and lighting devices. Developing materials to replace indium tin oxide (ITO) is accelerated from the viewpoint of cost and resources. We developed organic conductive films based on poly (3,4-ethylenedioxythiophene)-poly(styrene sulfonate). The ITO-free organic transparent electrodes were applied to polymer organic light emitting diodes (OLEDs). Solutions of the conductive electrodes and light emitting materials were spin-coated successively on transparent substrates, followed by vacuum deposition of cathode, $\mathrm{LiF}$ and $\mathrm{Al}$. We found that the organic conductive films made a good electrical contact to metals, but poor contact (high resistance) with some organic conductors, e.g., conductive glues of conductive metal tapes. Making use of this property, we could construct OLEDs without patterning transparent electrodes (anode), unlike the widely used ITO electrodes which require patterning for device applications to avoid shortcircuit. Because electric insulation is automatically made with our films, the device fabrication process will be drastically simplified. [DOI: 10.1380/ejssnt.2014.57]
\end{abstract}

Keywords: Electrical transport (conductivity, etc.); Semiconducting films; Electroluminescence; Polymer thin films; Organic electronics

\section{INTRODUCTION}

Optoelectronic devices, i.e., organic light emitting diodes (OLEDs) [1-4], are generally fabricated on transparent electrodes, which are mostly indium tin oxide (ITO). Polymer OLEDs [5-7] have been investigated for large and low cost applications like light-emitting packages, posters, and wall-paper, since they will be produced by printing technology, for example, roll-to-roll process. For the low cost production, printable organic transparent electrodes should be developed to replace ITO. Poly (3,4-ethylenedioxythiophene)-poly(styrene sulfonate) (PEDOT:PSS) is known as a good organic conductor, and the conductivity is improved by adding "solvents with higher boiling point than water" [8]. Many organic transparent electrodes have been developed along this line. Kajii et al. reported the comparison of small molecular OLEDs with ITO anode and ethylene glycol-modified-PEDOT:PSS anode [9]. Fehse and coworkers modified PEDOT:PSS by adding dimethylsulfoxide (DMSO) and fabricated small-molecular OLEDs with efficiency better than or comparable to that of ITO devices [10]. Na et al. developed organic solar cells using DMSO-modified-PEDOT:PSS anode, and obtained the efficiency close to that of ITO devices [11]. They made comparison with the ITO on glass and ITO on poly(ethyleneterephthalate) (PET) substrates. Conductivity of ITO on glass substrates is as high as $10^{4} \mathrm{~S} / \mathrm{cm}$

\footnotetext{
*This paper was presented at the 12 th International Conference on Atomically Controlled Surfaces, Interfaces and Nanostructures (ACSIN-12) in conjunction with the 21st International Colloquium on Scanning Probe Microscopy (ICSPM21), Tsukuba International Congress Center, Tsukuba, Japan, November 4-8, 2013.

†Corresponding author: w.mizutani@aist.go.jp
}

because it can be annealed at high temperature. In the case of PET substrates, the conductivity of ITO is limited by the annealing temperature to $10^{3} \mathrm{~S} / \mathrm{cm}$. It is difficult for organic electrodes to compete with ITO on glass substrates, but there may be chances to compete with or overcome ITO by the performance on plastic substrates. In order to compensate for the low conductivity of organic films, hybridization with metal-grid is reported [12, 13]. Inorganic materials were investigated as OLED anode materials $[14,15]$. New materials like carbon nanotubes [16] and graphene [17] were introduced to the transparent electrodes, and as an anode for OLEDs, higher efficiency than ITO devices was demonstrated [18]. Of course it is desirable to accomplish higher performances, but our target is the low cost fabrication using wet processes. We are developing organic transparent electrodes also based on PEDOT:PSS modified by diethylene glycol. When the electrodes can be formed from the liquid, various wet processes like spin, gravure, spray, and wire-bar coating are applicable. We examined the properties of the spin-coated films and searched suitable applications of the material.

As the transparent anode of polymer OLEDs, it seemed that patterning the electrodes was necessary for constructing devices. We thus developed a direct deposition method and fabricated organic-anode OLEDs with the same structure as the ITO-anode device to compare the performance. Recently, we succeeded to construct OLEDs without patterning transparent anodes using newly found unique property of the conductive film. Although there are techniques for patterning organic electrodes, some investments will be required for the practical implementation. Our newly proposed structure does not need extra investment for patterning. We expect that the device fabrication cost could be reduced significantly by this technique.

There is a shortcoming of polymer multilayer formation by wet process. The layers wet-processed previously 
may be damaged if similar solvents are used to form overlayers. Lamination process is expected to be a way-out, because polymer films are bonded directly without solvents [19-22]. Our new technique is successfully applied to the lamination process.

\section{EXPERIMENTAL}

Organic conductive films were formed from an organic dispersion liquid (TC-07-S, Denshi Kako, Co., LTD) based on PEDOT:PSS, which was modified to form stable films covering the surface of glass plates and PET films.

Glass substrates (Micro slide glass, Matsunami) were cut to the size of $26 \mathrm{~mm} \times 45 \mathrm{~mm}$, ultrasonic cleaned in acetone and ethanol for $10 \mathrm{~min}$, respectively, and dried by the flow of $\mathrm{N}_{2}$ each time. Thickness of the glass is about $1.2 \mathrm{~mm}$.

The TC-07-S was spin-coated on glass substrates with rotational speed from $500 \mathrm{rpm}$ to $3000 \mathrm{rpm}$ for $30 \mathrm{~s}$, and baked on a hot-plate at $120^{\circ} \mathrm{C}$ for $8 \mathrm{~min}$. We did not make any patterns, i.e. the whole surface of the glass substrates was covered with the organic conductive films.

For patterned electrodes, a dispenser (SHOT mini 100, Musashi Engineering) system, that controls the threedimensional position and pressure of a syringe, with a nozzle tube of the inner diameter of $0.14 \mathrm{~mm}$ was used to draw lines of 1-3 mm wide transparent electrodes on glass substrates.

Thickness of the spin-coated films was measured using a stylus-type surface profiler (DEKTAK ${ }^{3} \mathrm{ST}$, sloan), which scanned over the lines scratched by tweezers. We measured the thickness at central part and peripheral areas of one substrate, and plotted average and variation.

Sheet resistance was measured by the four-terminal method using a current of $0.1 \mathrm{~mA}$. The measurement was conducted at the center of the substrate and calibrated by the commercial instruments.

Transparency was characterized by a spectrometer (USB4000, Ocean Optics Inc.) with a light source (DTMini-2, Ocean Optics) and a softwear (SpectraSuite, Ocean Optics Inc.). To reduce the noise, we operated the system under the conditions of $500 \mathrm{~ms}$ integration time and averaging 40 times. The acquired spectrum was averaged over the wavelength of $10 \mathrm{~nm}$.

Polymer OLEDs were fabricated on the organic electrodes. Firstly, PEDOT:PSS (P VP AI4083, Heraeus), a buffer layer as well as a hole transport layer, was spincoated on the organic conductive films with $4000 \mathrm{rpm}$ for $30 \mathrm{~s}$, and followed by baking at $110^{\circ} \mathrm{C}$ for $5 \mathrm{~min}$.

As an emissive layer for green light, chlorobenzen solution of poly(N-vinyl carbazole) (PVK) mixed with N,N'-diphenyl-N,N'-bis(3-methylphenyl)1,1-biphenyl-4,40-diamine, 2-(4-biphenylyl)-5-(4tertbutylphenyl)-1,3,4-oxadiazole and fac-tris (2phenylpyridine)iridium $\left(\operatorname{Ir}(\text { ppy })_{3}\right) \quad$ (hereafter referred to as PVK-mix) was spin-coated on the PEDOT:PSS covered substrate at $2500 \mathrm{rpm}$ for $30 \mathrm{~s}$, and annealed at $80^{\circ} \mathrm{C}$ for $10 \mathrm{~min}[23]$.

For blue light emission, poly(9,9-dioctylfluorene) (PFO) (American Dye Source Inc.) [24] was dissolved in toluene $(1 \mathrm{wt} \%)$ and spin-coated on the prepared substrate at $1300 \mathrm{rpm}$ for $30 \mathrm{~s}$, and annealed at $80^{\circ} \mathrm{C}$ for
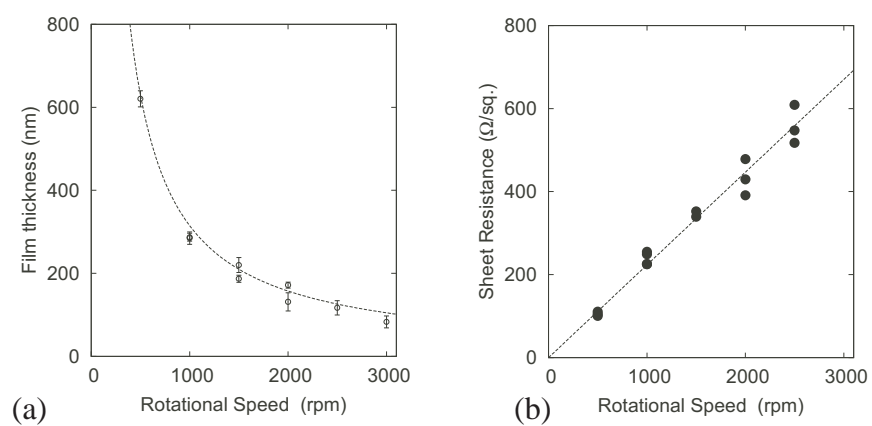

FIG. 1: Film thickness (a) and sheet resistance (b) were plotted as a function of spin-coating rotational speed (rms). Glass substrates $(26 \mathrm{~mm} \times 45 \mathrm{~mm})$ were used for spin-coating the conductive organic material for $30 \mathrm{~s}$. Data are fitted to simple curves with the fitting parameters $a$ and $b$, and plotted in dotted lines. (a) $d=a / x$, where $d$ is the thickness in nm, $x$ is rotational speed in rms, and $a=314864 \pm 4578$. (b) $R_{s}=b x$, where $R_{s}$ is sheet resistance in $\Omega /$ sq., and $b=0.223 \pm 0.004$.

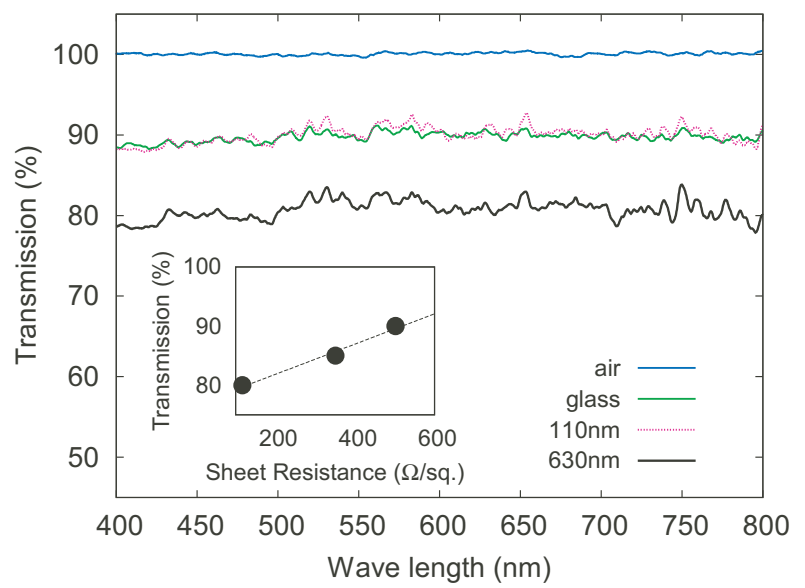

FIG. 2: Transmission of light through the organic electrodes. Inset: trade-off of the sheet resistance and the light transmission.

5 min.

We carried out the spin-coating and annealing processes under the atmospheric condition. Therefore, it is difficult for us to discuss the lifetime of the devices, since the following conditions are critical for the characterization [25]. For metal electrodes, $\mathrm{LiF}(0.8 \mathrm{~nm})$ and $\mathrm{Al}(70 \mathrm{~nm})$ were deposited on the polymer film through a shadow mask under high vacuum condition. Our vacuum deposition system is not equipped with the sample transfer to a glove-box, that is, the sample is exposed to air. Wiring to the devices was made using conductive aluminum tapes (AL-25BT, 3M) in a glove-box filled with dry $\mathrm{N}_{2}$.

For the lamination process, we used polyether sulfone (PES) (i-components) as a tentative substrate for cathode part. PVK-mix was spin-coated on PES at $2500 \mathrm{rpm}$ for $30 \mathrm{~s}$, and the cathode was deposited in vacuum. To apply heat and pressure to anode and cathode parts for lamination, we built a machine that presses $\mathrm{Cu}$ heater block to the films by solenoid.

The light emission and current were measured by a luminance meter (BM9, TOPCON) and an electric source meter (Keithley 2400). 


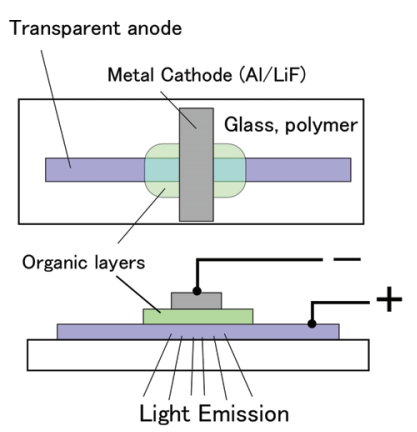

(a)

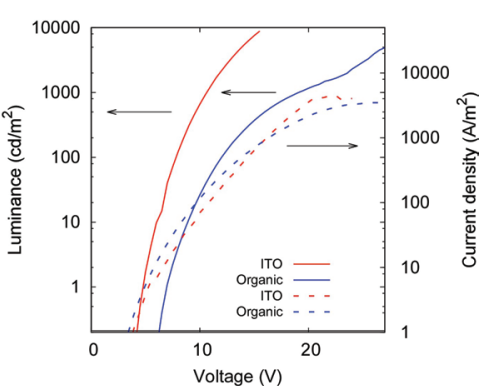

(b)

FIG. 3: (a) Device structure using anode patterned in line shape. The material sandwiched by anode and evaporated cathode emits light. Emission area was $2 \mathrm{~mm}$ by $2 \mathrm{~mm}$. (b) Luminance and current density of the ITO-anode device and organic-anode device as a function of voltage. The same structure as (a) and the same organic materials were used. Luminance curves are plotted in solid lines for ITO (red) and organic (blue) anode devices. Current density of the both devices is plotted in dotted line.

\section{RESULTS AND DISCUSSION}

\section{A. Basic properties of Organic Conductive Films}

We measured thickness, sheet resistance, and transparency of the organic conductive films spin-coated on glass. In general, we face a trade-off between conductivity and transparency.

\section{Thickness and sheet resistance of organic conducting films spin-coated on glass}

Figures 1(a) and (b) show the thickness and the sheet resistance of the organic conductive films as a function of the rotational speed of spin-coating. We found that the measured data could be fitted to simple functions like proportional and inversely proportional to the rotational speed, which was not self-apparent considering the possible precipitation of conductive materials at the interface, for example. From the fitting parameters $a$ and $b$ in Fig. 1, the sheet resistance $R_{s}=a b / d(\mathrm{~nm}) \simeq 70000 / d$. Resistivity $\rho$ of the films can be roughly derived by considering a square area as $\rho=10^{-2} \Omega \mathrm{cm}$. We checked the estimation experimentally using a line pattern deposited by the dispenser process, and obtained consistent result. Since the conductivity $\sigma=1 / \rho, \sigma$ is proportional to the thickness $d$, indicating that the conductive films should have uniform structure in terms of electric conduction.

\section{Transparency}

Transmission of light through the film on glass substrate was measured using a spectrometer as shown in Fig. 2. Glass substrates alone showed $90 \%$ transmission. We could not detect the difference in transmission from the glass substrate for the conductive films with the thickness less than $100 \mathrm{~nm}$ (sheet resistance larger than $550 \Omega /$ sq.), while we observed a large absorbance for the light with wavelength shorter than $380 \mathrm{~nm}$. For the films thicker than $250 \mathrm{~nm}$, the transmission smaller than the glass was measurable. The film with $630 \mathrm{~nm}$ on glass showed the transmission of about $80 \%$.

Sheet resistance can be reduced by increasing the thickness of the films, but the optical transparency decreases. As shown in the inset of Fig. 2, we have to compromise between the low sheet resistance $(\sim 110 \Omega /$ sq. $)$ and low transmission $(\sim 80 \%)$, and high resistance $(\sim 550 \Omega /$ sq. $)$ and high transmission $(\sim 90 \%)$.

\section{B. Applications of Organic Transparent Electrodes}

A line pattern with the same width $(2 \mathrm{~mm})$ of ITO line on glass was drawn on glass substrates using the dispenser system. Polymer OLED devices of the same structure as ITO test device were fabricated for comparison (Fig. 3(a)). As shown in Fig. 3(b), OLEDs using the organic anode showed the turn-on voltage of 8-9 V, which was higher by 1-2 $\mathrm{V}$ than that of the device using ITO. Apparently, extra voltage was needed to obtain the same luminance as the ITO device.

Luminance of the organic anode device shows steeper increase over the applied voltage of $20 \mathrm{~V}$. We speculate that this unexpected increase was caused by the crosssectional shape of the organic anode and resulting variance of the thickness of emitting layer. Thicker part may be more efficiently activated by the high voltage, and/or such emitting area grew larger at higher voltage. The current efficiency was $2.1 \mathrm{~cd} / \mathrm{A}$ at $4900 \mathrm{~cd} / \mathrm{m}^{2}$.

Besides the basic properties that we have shown in the previous subsections, we found a unique function of the conductive films. When a conductive $\mathrm{Al}$ tape was used to form electric contact with the conductive films, direct attachment did not make a good electric contact (Fig. 4(a)), namely, a large contact resistance was observed. The resistance value depended on the size of the contact area. Conductive tape of about $5 \mathrm{~mm}$ by $5 \mathrm{~mm}$ often showed fluctuation from about $100 \mathrm{k} \Omega$ to $10 \mathrm{M} \Omega$. To establish good contacts with the conductive metal tapes, a piece of metal foil needed to be inserted between the tape and the conductive film as shown in Fig. 4(b). Since we do not have evidences to prove the mechanism yet, we just speculate that the conductive adhesive material on the back side of the metal tape may have a band structure with energy level different from that of our conductive films. 


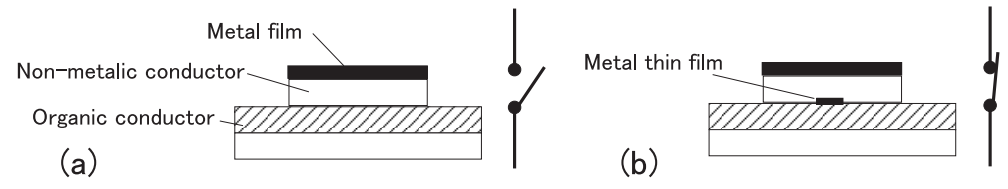

FIG. 4: Switching electrical conduction depending on the contact materials. (a) Metal tape directly contacting organic conductive film showed high resistance, while (b) sandwiched metal thin film reduced resistance significantly.

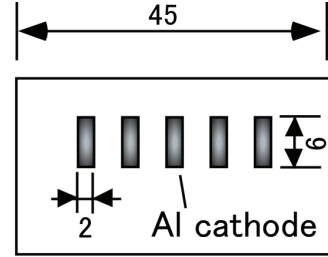

(a)

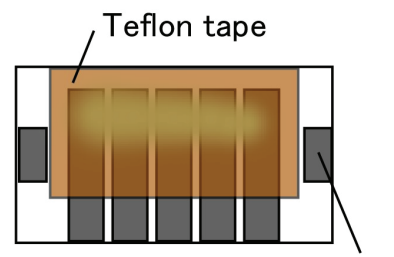

Anode contact

(d)

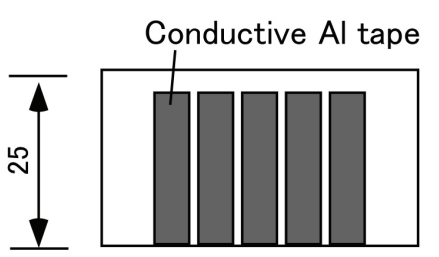

(b)

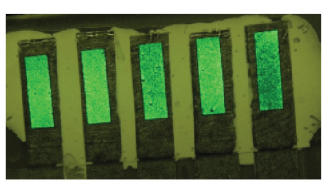

(e)

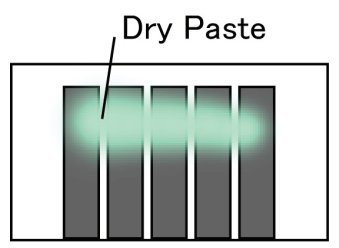

(c)

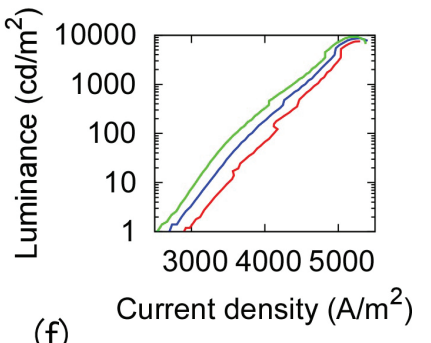

FIG. 5: OLED structure with patternless organic anode. (a) Whole surface of glass substrate is coated with the organic electrode, hole-transport layer, and polymer light emitting layer by spin-coating. Cathodes of $\mathrm{LiF}$ and $\mathrm{Al}$ are deposited on the organic layer through shadow mask under vacuum. (b) Conductive Al tapes are used to supply voltages to the cathode. (c) Desiccant paste (DryPaste, SAES Getters) was applied on the cathodes over the metal tapes. (d) Teflon tape was used to cover the devices to keep out humidity. Anode contacts were made at both sides of the devices to avoid the voltage drop in the substrate. (e) Photo of PVK-mix OLEDs emitting green light. Glass substrate covered with the organic anode film of about $250 \Omega /$ sq. was used. The photograph was taken after the performance of all the devices were measured, thus degraded by the operation. Black spots were observed as enlarged degradation around defects and contaminants. (f) Luminance-current density characteristics of three PVK-mix devices on the same substrate showing the maximum luminance of $10000 \mathrm{~cd} / \mathrm{m}^{2}$.

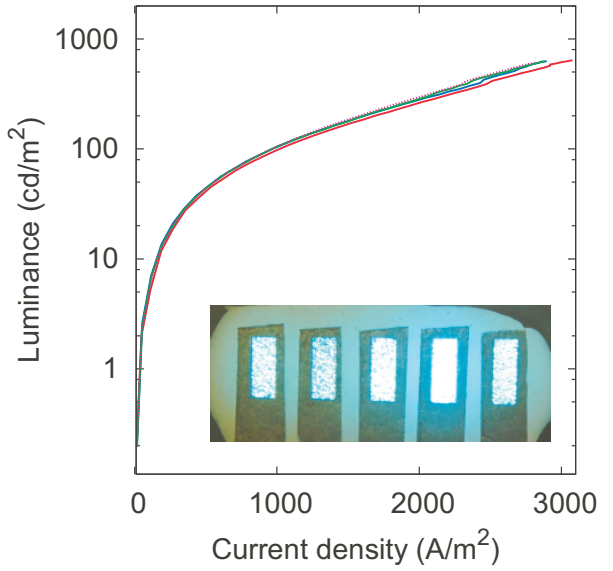

FIG. 6: Characteristics of three PFO OLED devices on the same substrate. Organic anode film of $200 \mathrm{~nm}$ thick was spincoated on glass substrate. Inset: Photograph of five PFO devices emitting blue light. Since the photograph was taken after the measurements, black spots were prominent as the sign of degradation.

Since the energy level of metals is continuous, the current may flow through the inserted metal foil. Another possible explanation is that a large barrier formed between the different materials may hinder the current flow. The metal piece can be gold foils or evaporated aluminum films working as a cathode of OLED devices.

\section{OLED using patternless anode}

We propose a simple OLED structure using the organic transparent electrode which is not patterned as shown in Fig. 5. We only need to pattern the metal cathodes which define the light emitting area. After spin-coating organic layers, we evaporated aluminum through a shadow mask in vacuum (Fig. 5(a)). In the case of OLED devices using ITO anode, metal wires applying negative voltage to the cathode need to be insulated from the anode to avoid short-circuit. ITO anodes are thus patterned so that they are separated from the cathode lines. However, in the area where the organic anode and the conductive tapes contacted directly without metal films, the insulation, or high contact resistance state, is automatically attained. This automatic insulation was also observed on the area covered with the emissive layer, although the contact resistance values slightly decreased depending on the layer properties, resulted in an increase in the current.

Figures 5(c) and (d) show the sealing of the devices, and Figs. 5(e) and (f) demonstrate the performance of PVK-mix devices with this structure. For the operation, we applied negative voltages to the conductive $\mathrm{Al}$ tape, and the electron should flow through the conduc- 


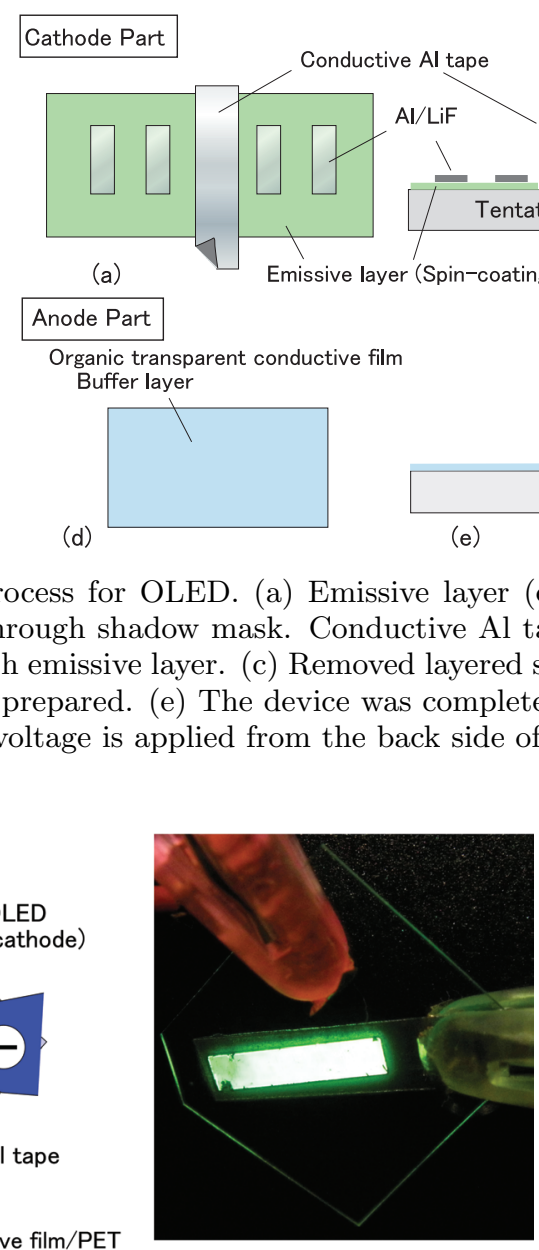

FIG. 8: Laminated OLED using PVK-mix. Maximum luminance of $\sim 350 \mathrm{~cd} / \mathrm{m}^{2}$ at $13 \mathrm{~V}$ was obtained. Part of the $\mathrm{Al}$ tape directly contacted to the conductive film does not cause short-circuit.
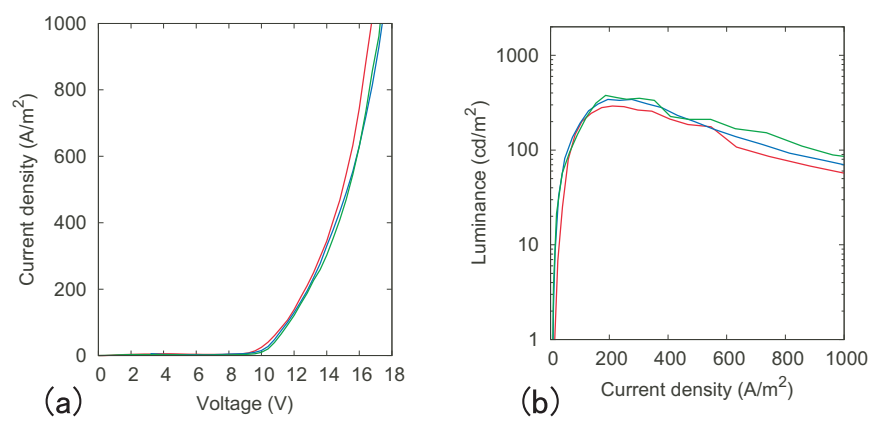

FIG. 9: Performances of laminated OLED using PVK-mix. (a) Current density - voltage characteristics and (b) luminance current density characteristics.

tive glue via cathode metals to the organic anode. We found that the resistance between the organic anode and the conducting tape decreased by sandwiched PVK-mix film, and as a result, larger leak current should have flowed in the area where the tape contacted without metal films, showing the decrease in the current efficiency $(1.8 \mathrm{~cd} / \mathrm{A})$. The luminance-current density characteristics deformed because of this unwanted current increase. The increase of the leak current and resulting deformation were not observed for PVK-mix devices using the conventional structure as shown in Fig. 3(b). There are limps in charac- teristics, which were caused by steps in current-voltage curves. Because we did not observe the corresponding change in luminance-voltage curves, the steps in the current must be caused in the leak current component. Since we needed to apply voltages as high as $30 \mathrm{~V}$ to obtain the maximum luminance for these devices, the high voltages may have caused small damages at limited parts in the direct contact area.

Figure 6 shows the characteristics of three PFO devices [24] fabricated on the same substrate covered with the spin-coated organic anode. The same process as Figs. 5(a)-(d) was repeated. We obtained the maximum luminance as large as $500 \mathrm{~cd} / \mathrm{m}^{2}$ at $3000 \mathrm{~A} / \mathrm{m}^{2}$. The PFO devices showed the turn-on voltage of $4.5 \mathrm{~V}$, which is about half of the PVK-mix devices.

\section{Lamination process}

Guo et al. demonstrated that the polymer OLEDs can be constructed by lamination process [19]. The proposed OLED structure can be fabricated by lamination process as shown in Fig. 7. We used PET substrates with the gravure coated organic conductive films with a sheet resistance of $170 \Omega /$ sq. The emitting layer was spin-coated on PES substrate, and the cathode metal was evaporated on the layer (Fig. 7(a)). A strip of the Al tape was pasted over the cathode part, and removed the cathode with the emissive layer(Fig. 7(b)). The tape was then laminated on the PET with the organic anode (Fig. 7(e)) under the heat and pressure. Lamination conditions that we used were $110-150{ }^{\circ} \mathrm{C}$ and $\sim 2 \mathrm{MPa}$ for $10 \mathrm{sec}$.

An example of the PVK-mix OLED device fabricated by lamination process is shown in Fig. 8 .

Figure 9 shows performances of laminated three PVKmix devices. The shape of luminance - current density characteristics was not like those of the devices fabricated without lamination (Fig. 5(f)), indicating that the leak current was not dominant in the PVK-mix laminated devices. 


\section{CONCLUSION}

From the PEDOT:PSS based dispersion liquid, conductive films with the thickness of 100-600 nm, sheet resistance of $120-500 \Omega /$ sq., and transmission of $80-90 \%$ can be obtained by spin-coating on glass substrates. The resistivity of about $10^{2} \Omega \cdot \mathrm{m}$ was derived from the measurements of thickness and sheet resistance. The conductivity of the films were found to be proportional to the film thickness, indicating the uniform structure of the films. We found that contact resistance to the organic film was high un- less the contacting material is metal. Polymer OLEDs using patternless organic transparent conductive films as anode were proposed and demonstrated. Because the insulation between the organic anode and the metal tapes is automatically made, the patterning of anode is not necessary and the electrical connection to the cathodes is simplified, although we found that the contact resistance could decrease when PVK-mix layer was sandwiched. The proposed structure should reduce the production cost of polymer OLEDs, and open up novel applications.
[1] C. W. Tang and S. A. VanSlyke, Appl. Phys. Lett. 51, 913 (1987).

[2] J. Kido, K. Hongawa, K. Okuyama, and K. Nagai, Appl. Phys. Lett. 64, 815 (1994).

[3] Y. Hino, H. Kajii, and Y. Ohmori, Organic Electronics 5, 265 (2004).

[4] Y. Hino, H. Kajii, and Y. Ohmori, Jpn. J. Appl. Phys. 44, 2790 (2005).

[5] R. H. Friend, R. W. Gymer, A. B. Holmes, J. H. Burroughes, R. N. Marks, C. Taliani, D. D. C. Bradley, D. A. D. Santos, J. L. Bredas, M. Logdlund, and W. R. Salaneck, Nature 397, 121 (1999).

[6] H. Wu, J. Zou, F. Liu, A. Mikhailovsky, G. C. Bazan, W. Yang, and Y. Cao, Adv. Mater. 20, 696 (2008).

[7] J.-H. Choi, K.-H. Kom, S.-J. Choi, and H. H. Lee, Nanotechnology 17, 2246 (2006).

[8] J. Kim, J. Jung, D. Lee, and J. Joo, Synth. Met. 126, 311 (2002).

[9] H. Kajii, Y. Sekimoto, H. Maki, and Y. Ohmori, IEICE Gen. Conf. '07 CS-9-3 (2007).

[10] K. Fehse, K. Walzer, K. Leo, W. Lovenich, and A. Elschner, Adv. Mater. 19, 441 (2007).

[11] S.-I. Na, S.-S. Kim, J. Jo, and D.-Y. Kim, Adv. Mater. 20, 4061 (2008)

[12] J. Zou, H.-L. Yip, S. K. Hau, and A. K.-Y. Jen, Appl. Phys. Lett. 96, 203301 (2010).

[13] H. Lee, S.-W. Seo, E. Jung, H. Chae, and S. M. Cho, Appl. Phys. Express 6, 046503 (2013).
[14] L. Zugang, Z. Weiming, J. Rongbin, Z. Zhili, J. Xueyin, X. Minzhao, and F. Bin, J. Phys.: Condens. Matter 8, 3221 (1996).

[15] H. Kim, J. S. Horwitz, W. H. Kim, A. J. Mäkinen, Z. H. Kafafi, and D. G. Chrisey, Thin Solid Films 420-421, 539 (2002).

[16] M. W. Rowell, M. A. Topinka, M. D. McGehee, H.-J. Prall, G. Dennler, N. S. Sariciftci, L. Hu, and G. Gruner, Appl. Phys. Lett. 88, 233506 (2006).

[17] G. Eda, Y.-Y. Lin, S. Miller, C.-W. Chen, W.-F. Su, and M. Chhowalla, Appl. Phys. Lett. 92, 233305 (2008).

[18] T.-H. Han, Y. Lee, M.-R. Choi, S.-H. Woo, S.-H. Bae, B. H. Hong, J.-H. Ahn, and T.-W. Lee, Nature Photonics 6, 105 (2012).

[19] T.-F. Guo, S. Pyo, S.-C. Chang, and Y. Yang, Adv. Func. Mater. 11, 339 (2001).

[20] T.-W. Lee, J. Zaumseil, Z. Bao, J. W. P. Hsu, and J. A. Rogers, Proc. Natl. Acad. Sci. U. S. A. 101, 429 (2004).

[21] J. Liu, L. N. Lewis, T. J. Faircloth, and A. R. Duggal, Appl. Phys. Lett. 88, 223509 (2006).

[22] J. Huang, G. Li, and Y. Yang, Adv. Funct. Mater. 20, 415 (2008).

[23] D.-H. Lee, H. Chae, and S. M. Cho, Jpn. J. Appl. Phys. 48, 060204 (2009).

[24] D. Neher, Macromol. Rapid. Commun. 22, 1365 (2001).

[25] K. Fehse, R. Meerheim, K. Walzer, K. Leo, and a. A. E. Wilfried Lövenich, Appl. Phys. Lett. 93, 083303 (2008). 